\title{
A!
}

This is an electronic reprint of the original article.

This reprint may differ from the original in pagination and typographic detail.

Huttunen, Mikko J.; Mäkitalo, Jouni; Bautista, Godofredo; Kauranen, Martti

\section{Multipolar nonlinear light-matter interactions with Gaussian vector beams}

Published in:

Nonlinear Optics and Its Applications VIII; and Quantum Optics III

DOI:

$10.1117 / 12.2052307$

Published: 01/01/2014

Document Version

Publisher's PDF, also known as Version of record

Please cite the original version:

Huttunen, M. J., Mäkitalo, J., Bautista, G., \& Kauranen, M. (2014). Multipolar nonlinear light-matter interactions with Gaussian vector beams. In B. J. Eggleton, A. L. Gaeta, N. G. R. Broderick, A. V. Sergienko, A.

Rauschenbeutel, \& T. Durt (Eds.), Nonlinear Optics and Its Applications VIII; and Quantum Optics III (Vol. 9136). [91361R] (Proceedings of SPIE : the International Society for Optical Engineering; Vol. 9136). SPIE.

https://doi.org/10.1117/12.2052307

This material is protected by copyright and other intellectual property rights, and duplication or sale of all or part of any of the repository collections is not permitted, except that material may be duplicated by you for your research use or educational purposes in electronic or print form. You must obtain permission for any other use. Electronic or print copies may not be offered, whether for sale or otherwise to anyone who is not an authorised user. 


\section{Multipolar nonlinear light-matter interactions with Gaussian vector beams}

Mikko J. Huttunen, Jouni Mäkitalo, Godofredo Bautista, Martti Kauranen

Mikko J. Huttunen, Jouni Mäkitalo, Godofredo Bautista, Martti Kauranen, "Multipolar nonlinear light-matter interactions with Gaussian vector beams," Proc. SPIE 9136, Nonlinear Optics and Its Applications VIII; and Quantum Optics III, 91361R (1 May 2014); doi: 10.1117/12.2052307

SPIE. Event: SPIE Photonics Europe, 2014, Brussels, Belgium 


\title{
Multipolar nonlinear light-matter interactions with Gaussian vector beams
}

\author{
Mikko J. Huttunen ${ }^{*, a, b}$, Jouni Mäkitalo ${ }^{\text {a }}$, Godofredo Bautista ${ }^{a}$, Martti Kauranen ${ }^{a}$ \\ ${ }^{a}$ Department of Physics, Tampere University of Technology, P.O. Box 692, FI-33101 Tampere, \\ Finland \\ ${ }^{\mathrm{b}}$ COMP Centre of Excellence, Department of Applied Physics, Aalto University, P.O. Box 15100, \\ FI-00076 Aalto, Finland
}

\begin{abstract}
We show that surface second-harmonic generation (SHG) with focused Gaussian vector beams can be described in terms of effective Mie-type multipolar contributions to the SHG signal even in the electric dipole approximation of constitutive relations. Traditionally, Mie-type multipoles arise from field retardation across nanoparticles. In our case, the multipolar light-matter interaction is due to excitation with Gaussian vector beams and the tensorial properties of the SH response. As different multipoles have different radiative properties, we demonstrate the presence of multipoles by measuring strongly asymmetric SH emission into reflected and transmitted directions from a nonlinear thin film with isotropic surface symmetry, where symmetric emission is expected using traditional formalisms based on plane-wave excitation. The proposed multipole approach provides a convenient way to explain the measured asymmetric emission. Secondly, we generalize the treatment beyond the electric dipole approximation and propose that analogous vector excitationinduced multipolar effects could also occur in the microscopic light-matter interaction. Our results may allow new possibilities to designing confined and thin nonlinear sources with desired radiation patterns.
\end{abstract}

Keywords: Light-matter interaction, Multipoles, Mie scattering, Nonlinearities, Second-harmonic generation,

\section{INTRODUCTION}

The optical responses of materials are in general described by convolutional integrals of the non-local material response function and the total field, causing mathematical complications ${ }^{1}$. A common simplification is to use an approximation where only electric-dipole (ED) interactions between light and matter are considered, as the higher multipole contributions to the interaction are usually much weaker. However such multipole contributions, for example due to magnetic dipoles and electric quadrupoles, are needed to explain several optical phenomena, such as optical activity of chiral materials or selection rules in spectroscopy ${ }^{2}$.

In nanostructured materials, multipolar effects can arise from two different sources. The first originates from the atomiclevel description of the light-matter interaction (LMI) with the corresponding Hamiltonians ${ }^{2}$. The second is purely macroscopic in origin and arises from the Mie solutions to the Maxwell's equations where effective multipoles are due to retardation of the excitation field in the presence of finitely-sized nanoparticles ${ }^{3,4}$. The multipolar contributions can become considerable especially when the spatial dimensions of the material constituents are comparable to wavelength, as some recent nonlinear studies have shown ${ }^{5-8}$. But better understanding and mathematical methods for such interactions is still needed to properly utilize them in applications, for example in enhancing the directionality of optical antennas ${ }^{9,10}$.

Multipolar LMIs are also important in nonlinear optics (NLO), since second-order effects are forbidden from centrosymmetric materials within the ED approximation ${ }^{11}$. Second-order effects, such as second-harmonic generation (SHG), can occur in centrosymmetric bulk materials only due to weak magnetic or quadrupolar contributions, in practice restricting the possible materials for NLO. On the other hand, second-order effects are always allowed at interfaces and surfaces $^{12}$, which has made the separation of these ED allowed surface contributions from the multipolar bulk

"mikko.huttunen@aalto.fi; phone +35850 4921272

Nonlinear Optics and Its Applications VIII; and Quantum Optics III, edited by Benjamin J. Eggleton, Alexander L. Gaeta, Neil G. R. Broderick, Alexander V. Sergienko, Arno Rauschenbeutel, Thomas Durt, Proc. of SPIE Vol. 9136, 91361R - (c) 2014 SPIE · CCC code: 0277-786X/14/\$18 - doi: 10.1117/12.2052307 
contributions extremely difficult. Only recently, the separation has been performed in an unambiguous and quantitative way showing hope for the future ${ }^{13,14}$.

In this paper, we show how ED-allowed effective multipolar effects can arise when focused vector beam excitation is used. We measure strongly asymmetric transmitted and reflected SHG (5-fold difference) from a thin silicon nitride (SiN) sample, where traditional scalar treatment predicts symmetric emission. We interpret the result in terms of Mietype multipolar effects, which originate from the focused vector excitation. We show how higher-order Gaussian beams could be used to engineer the demonstrated multipolar effects even in the weak focusing regime. Finally, we generalize our treatment to microscopic LMI and propose that similar multipolar effects could also occur for samples closer to point-like sources.

\section{THEORY}

\subsection{Mie-type Multipoles due to spatially confined excitation}

We start our theoretical treatment by writing the second-harmonic (SH) source polarization for a single frequency $\omega$ in a general convolutional form ${ }^{15}$

$$
\mathbf{P}(\mathbf{r})=\varepsilon_{0} \iint_{-\infty}^{\infty} \chi^{(2)}\left(\mathbf{r}-\mathbf{r}_{1}, \mathbf{r}-\mathbf{r}_{2}\right): \mathbf{E}\left(\mathbf{r}_{1}\right) \mathbf{E}\left(\mathbf{r}_{2}\right) \mathrm{d}^{2} \mathbf{r}_{1} \mathrm{~d}^{2} \mathbf{r}_{2},
$$

where $\mathbf{E}$ is the total field at the fundamental frequency $\omega / 2, \chi^{(2)}$ is the second-order susceptibility tensor and $\mathbf{r}=(\mathrm{x}, \mathrm{y}, \mathrm{z})$ is position vector in Cartesian coordinates. We restrict our treatment to surfaces only, and define the $x y$-plane of interest by $z=0$. In the ED approximation, $\chi^{(2)}$ is local, i.e. independent of $\mathbf{r}_{1}$ and $\mathbf{r}_{2}$, and Eq. (1) simplifies into

$$
\mathbf{P}(\mathbf{r})=\varepsilon_{0} \chi^{(2)}(\mathbf{r}): \mathbf{E}(\mathbf{r}) \mathbf{E}(\mathbf{r}) .
$$

This form is traditionally used in NLO, and next we show how effective multipoles can arise also in this description when the spatially-varying incident field is properly considered ${ }^{16,17}$. We follow our recently proposed approach ${ }^{18}$, and assume that the source polarization can be expressed using Helmholtz decomposition ${ }^{1}$

$$
\mathbf{P}(\mathbf{r})=\mathbf{P}_{\mathrm{i}}+\nabla \times \mathbf{P}_{\mathrm{s}},
$$

where $\mathbf{P}_{\mathrm{i}}$ is the irrotational (curl-free) and $\nabla \times \mathbf{P}_{\mathrm{S}}$ is the solenoidal (divergence-free) part. This Eq. (3) provides understanding for our experimental results as the symmetry properties of the two terms are different ${ }^{1}$. Therefore, the different terms can give rise to interference effects (e.g. directionality or asymmetry) in the emission, and it can also be shown that the solenoidal part is needed to obtain NLO responses of magnetic character ${ }^{18}$.

The next step is to introduce some means to control the relative strength of the non-zero solenoidal term in the source polarization of Eq. (3). One approach would be to use Hermite-Gaussian (HG) or Laguerre-Gaussian (LG) beams of different orders. Different modes can be generated from the fundamental beam $\mathbf{E}_{00}^{\mathrm{HG}}(\mathbf{r})$ by using ${ }^{19,20}$

$$
\mathbf{E}_{n m}^{\mathrm{HG}}(\mathbf{r})=w_{0}^{n+m} \frac{\partial^{n}}{\partial x^{n}} \frac{\partial^{m}}{\partial y^{m}} \mathbf{E}_{00}^{\mathrm{HG}}(\mathbf{r}),
$$

for HG beams where $n$ and $m$ denote the different order of the beam and $w_{0}$ is beam waist. Similarly, LG beams can be generated using

$$
\mathbf{E}_{p l}^{\mathrm{LG}}(\mathbf{r})=k^{p} w_{0}^{2 p+l} \mathrm{e}^{-i k z} \frac{\partial^{p}}{\partial z^{p}}\left(\frac{\partial}{\partial x}+i \frac{\partial}{\partial y}\right)^{l}\left[\mathbf{E}_{00}^{\mathrm{LG}}(\mathbf{r}) \mathrm{e}^{-i k z}\right],
$$

where $k$ is the wavenumber and $\mathbf{E}_{00}^{\mathrm{LG}}(\mathbf{r})$ is the fundamental LG mode. Since fundamental modes are even in both $x$ and $y$, the next higher mode $\mathbf{E}_{11}^{\mathrm{HG} / \mathrm{LG}}(\mathbf{r})$ will be odd in $x$ and $y$ and so on. Therefore, two beams with properly chosen mode orders could be utilized to engineer the solenoidal term. 
Our approach follows a similar idea, but is simpler to demonstrate experimentally. We use strongly focused vector Gaussian beams, where the localization of light at the focal plane gives rise to relatively strong longitudinal field ${ }^{19}$. The longitudinal field also necessarily has different symmetry than the transverse components. This can be seen by assuming a fundamental $x$-polarized HG beam under paraxial approximation and applying zero divergence condition for the field. Then we get ${ }^{19}$

$$
E_{z}(\mathbf{r})=-\int \frac{\partial E_{x}(\mathbf{r})}{\partial x} \mathrm{~d} z
$$

From Eq. (6) one can see that if the transverse $x$-polarized part is even in $x$ then the longitudinal part is odd. The opposite would occur if a next higher-order mode were used. Similar argumentation holds also beyond paraxial approximation when the fields are more rigorously calculated using the angular spectrum representation ${ }^{19}$. Therefore, a non-zero solenoidal term is expected also when using focused vector Gaussian beam excitation, provided that the second-order susceptibility contains tensor components, which depend on both $\mathrm{x}$ and $\mathrm{z}$ field components and preserve the asymmetry. Perhaps the simplest sample with the right kind of susceptibility tensor is an isotropic surface sample which has non-zero susceptibility components of $z z z, z x x=z y y$ and $x x z=x z x=y y z=y z y$. The $z z z$ and $z x x=z y y$ contribute to the irrotational and $x x z=x z x=y y z=y z y$ contribute to the solenoidal terms in $\mathbf{P}(\mathbf{r})$ giving rise to directional SHG even from a thin sample.

\subsection{Microscopic multipoles due to the spatially confined excitation}

In the previous section we showed how multipolar effects can occur in the ED-allowed LMI, and in the next section we show the related experimental results. But the treatment corresponded to an effectively macroscopic picture, where the excitation field interacts with a sample of macroscopic size and multipolar effects occur. Now we return to the general form of Eq. (1), which describes the non-local second-order polarization $\mathbf{P}(\mathbf{r})$ at point $\mathbf{r}$. We term this picture as a microscopic picture of LMI, where multipolar effects occur due to the non-locality of the LMI. Next we show how our approach can also provide insight to this microscopic picture.

Traditionally the non-local responses of Eq. (1) are treated by taking a series expansion and dividing the integral into various multipolar terms, which have well-defined symmetry properties with respect to parity (oddness/evenness) and time (symmetric/anti-symmetric) and radiate accordingly ${ }^{1}$. The different radiation properties also make possible to separate the contributions from various terms, as has been demonstrated using metal nanoparticles ${ }^{21}$ as well as bulk materials ${ }^{22}$. But equally correct approach would be to numerically calculate the non-local polarization directly using Eq. (1). Such an approach can be made numerically more efficient by using Fourier transforms (FTs) and convolution theorem in the spatial frequency domain ${ }^{15}$. As the derivation of the spatial frequency domain description of polarization in Ref. [15] is quite compact, we now show the full derivation. We start by using the commutation property of the convolution to write Eq. (1) as

$$
\mathbf{P}(\mathbf{r})=\varepsilon_{0} \iint_{-\infty}^{\infty} \chi^{(2)}\left(\mathbf{r}_{1}, \mathbf{r}_{2}\right): \mathbf{E}\left(\mathbf{r}-\mathbf{r}_{1}\right) \mathbf{E}\left(\mathbf{r}-\mathbf{r}_{2}\right) \mathrm{d}^{2} \mathbf{r}_{1} \mathrm{~d}^{2} \mathbf{r}_{2},
$$

to emphasize that in our approach the non-locality of the LMI is contained in the E-field distributions, and that the susceptibility is kept local. Next, we define the FT and inverse FT of the incident field as

$$
\begin{aligned}
& \mathbf{E}(\mathbf{r})=\int_{-\infty}^{\infty} \hat{\mathbf{E}}\left(k_{x}, k_{y}, z\right) \mathrm{e}^{\mathrm{i} \mathbf{k} \cdot \mathbf{r}} \mathrm{d}^{2} \mathbf{k}, \\
& \widehat{\mathbf{E}}\left(k_{x}, k_{y}, z\right)=\frac{1}{4 \pi^{2}} \int_{-\infty}^{\infty} \mathbf{E}(\mathbf{r}) \mathrm{e}^{-\mathrm{i} \mathbf{k} \cdot \mathbf{r}} \mathrm{d}^{2} \mathbf{r},
\end{aligned}
$$

where $\mathbf{k}=\left(k_{x}, k_{y}, k_{z}\right)$ is the wave vector and double integrations over $\left(k_{x}, k_{y}\right)$ and $(x, y)$ are implied. Using Eq. (8) and the translation property of FT we get

$$
\mathbf{P}(\mathbf{r})=\varepsilon_{0} \iint_{-\infty}^{\infty} \iint_{-\infty}^{\infty} \mathrm{e}^{-\mathrm{i} \mathbf{k}_{1} \cdot \mathbf{r}_{1}} \mathrm{e}^{-\mathrm{i} \mathbf{k}_{2} \cdot \mathbf{r}_{2}} \chi^{(2)}\left(\mathbf{r}_{1}, \mathbf{r}_{2}\right): \hat{\mathbf{E}}\left(k_{x, 1}, k_{y, 1}, 0\right) \hat{\mathbf{E}}\left(k_{x, 2}, k_{y, 2}, 0\right) \mathrm{e}^{\mathrm{i}\left(\mathbf{k}_{1}+\mathbf{k}_{2}\right) \cdot \mathbf{r}} \mathrm{d}^{2} \mathbf{r}_{1} \mathrm{~d}^{2} \mathbf{r}_{2} \mathrm{~d}^{2} \mathbf{k}_{1} \mathrm{~d}^{2} \mathbf{k}_{2},
$$

which we can simplify by defining 


$$
\hat{\chi}^{(2)}\left(\mathbf{k}_{1}, \mathbf{k}_{2}\right)=\iint_{-\infty}^{\infty} \mathrm{e}^{-\mathrm{i} \mathbf{k}_{1} \cdot \mathbf{r}_{1}} \mathrm{e}^{-\mathrm{i} \mathbf{k}_{2} \cdot \mathbf{r}_{2}} \chi^{(2)}\left(\mathbf{r}_{1}, \mathbf{r}_{2}\right) \mathrm{d}^{2} \mathbf{r}_{1} \mathrm{~d}^{2} \mathbf{r}_{2}
$$

into form

$$
\mathbf{P}(\mathbf{r})=\varepsilon_{0} \iint_{-\infty}^{\infty} \hat{\chi}^{(2)}\left(\mathbf{k}_{1}, \mathbf{k}_{2}\right): \hat{\mathbf{E}}\left(k_{x, 1}, k_{y, 1}, 0\right) \hat{\mathbf{E}}\left(k_{x, 2}, k_{y, 2}, 0\right) \mathrm{e}^{\mathrm{i}\left(\mathbf{k}_{1}+\mathbf{k}_{2}\right) \cdot \mathbf{r}} \mathrm{d}^{2} \mathbf{k}_{1} \mathrm{~d}^{2} \mathbf{k}_{2} .
$$

To perform the integral we take a FT of the source polarization of Eq. (12) and perform the integration over $\mathbf{r}$

$$
\begin{aligned}
\widehat{\mathbf{P}}(\mathbf{k}) & =\frac{\varepsilon_{0}}{4 \pi^{2}} \iiint_{-\infty}^{\infty} \hat{\chi}^{(2)}\left(\mathbf{k}_{1}, \mathbf{k}_{2}\right): \hat{\mathbf{E}}\left(k_{x, 1}, k_{y, 1}, 0\right) \hat{\mathbf{E}}\left(k_{x, 2}, k_{y, 2}, 0\right) \mathrm{e}^{\mathrm{i}\left(\mathbf{k}_{1}+\mathbf{k}_{2}-\mathbf{k}\right) \cdot \mathbf{r}} \mathrm{d}^{2} \mathbf{k}_{1} \mathrm{~d}^{2} \mathbf{k}_{2} \mathrm{~d}^{2} \mathbf{r}, \\
& =\frac{\varepsilon_{0}}{2 \pi} \iint_{-\infty}^{\infty} \hat{\chi}^{(2)}\left(\mathbf{k}_{1}, \mathbf{k}_{2}\right): \hat{\mathbf{E}}\left(k_{x, 1}, k_{y, 1}, 0\right) \hat{\mathbf{E}}\left(k_{x, 2}, k_{y, 2}, 0\right) \delta\left(\mathbf{k}_{1}+\mathbf{k}_{2}-\mathbf{k}\right) \mathrm{d}^{2} \mathbf{k}_{1} \mathrm{~d}^{2} \mathbf{k}_{2} .
\end{aligned}
$$

The final form is gotten by integrating Eq. (14) into

$$
\widehat{\mathbf{P}}(\mathbf{k})=\varepsilon_{0} \chi^{(2)}\left(\mathbf{k} ; \mathbf{k}_{1}, \mathbf{k}_{2}\right): \widehat{\mathbf{E}}\left(k_{x, 1}, k_{y, 1}, 0\right) \widehat{\mathbf{E}}\left(k_{x, 2}, k_{y, 2}, 0\right),
$$

where the notation of the arguments in $\chi^{(2)}\left(\mathbf{k} ; \mathbf{k}_{1}, \mathbf{k}_{2}\right)$ reflects the conservation of momentum $\mathbf{k}=\mathbf{k}_{1}+\mathbf{k}_{2}$ in the process. The mathematical form is completely analogous to the traditional form for second-order source polarization in ED approximation [see Eq. (2)]. Therefore, as effective multipolar effects in ED approximation can occur solely due to the vector excitation ${ }^{18}$, Eq. (15) predicts that analogous non-local/multipolar effects should occur in the spatial frequency description. Further work is needed to appropriately design the structure of $\chi^{(2)}\left(\mathbf{k} ; \mathbf{k}_{1}, \mathbf{k}_{2}\right)$ for the desired emission pattern of SHG.

\section{RESULTS}

The experimental setup for measuring the transmitted and reflected SHG is described elsewhere ${ }^{18}$. Briefly, a fs-laser with central wavelength of $1060 \mathrm{~nm}$ was focused onto the thin films $(\sim 50 \mathrm{~nm})$ of silicon nitride (SiN). The numerical aperture (NA) of the focusing objective was 0.8 . The SiN was positioned at the focal plane and reflected and transmitted SHG was collected using the focusing objective and another identical objective at the backside of the sample, respectively. As we were interested in the relative strengths between the transmitted and reflected emissions, the collection efficiencies in both detection arms were calibrated. SHG beams were filtered from the fundamental beam and measured using photomultiplier tubes connected to a photon counting unit.

Simulations were performed using Green's function approach for SHG described earlier ${ }^{23}$. To provide stronger evidence for the effect, we measured and simulated the SHG responses while changing the polarization state of incident beam from linear to circular. The interference effects due to multipolar contributions were expected to be maximized for linear input polarization. Exactly this behavior was measured, as is seen in Fig. (1a), which shows the measured and simulated SHG signals both in transmitted and reflected directions. To estimate how strongly the used NA for the focusing objective affects the results, we simulated the total radiated power [see Fig.(1b)] and the ratio of the transmitted/reflected SHG power [see Fig. (1c)]. 

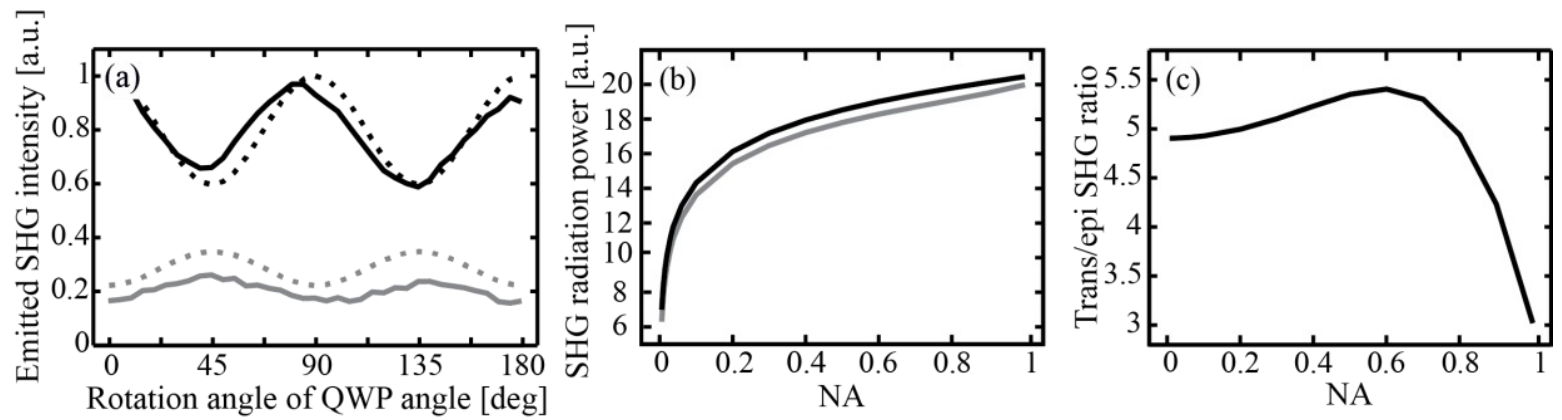

Figure 1. (a) Measured (solid) and simulated (dotted) line plots show clear $\sim 5$-fold difference between transmitted (black) and reflected (gray) SHG emission. Also the polarization-dependences of the signals are different. The transmitted (reflected) signal has maximum (minimum) when input polarization is linear (circular). (b) Simulated strength of the radiated SHG emission tends to zero when the NA of the focusing objective is decreased. Despite the total radiated SHG power greatly decreases when NA is decreased, (c) the ratio of the transmitted/reflected SHG emission does not tend to unity.

\section{DISCUSSION}

First, we exclude other possible origins for the asymmetric SHG emission. The thickness of the samples was less than $\lambda / 10$, and therefore possible phase-matching or Fabry-Perot issues cannot explain the asymmetry. Neither can absorption explain the effect, as the transmitted and reflected emission had also different polarization dependence. On the other hand, our calculations reproduce the polarization behavior perfectly. Neither can SHG from the glass substrate explain the effect, as we did not observe measurable SHG from the substrate even when the excitation power was tripled, which should correspond to 9-fold increase in SHG signal. Therefore, the most plausible explanation are the effective multipolar contributions due to the vectorial excitation.

Then we consider the benefits of our approach. The effective macroscopic multipoles [Eqs. (2) and (3)] are discussed in detail in elsewhere ${ }^{18}$, and therefore we concentrate on the microscopic LMI. Although the non-locality of fields is already visible after the commutation of Eq. (1), representing the source polarization in the spatial frequency domain provides benefits. Firstly, understanding some of the occurring physics is clearer as the integral form of the convolution is transformed into multiplication. The excitation field distributions correspond to propagating HG/LG modes, and hence we know that the functional form of the distributions remains unchanged in the FT. In addition, from the basic properties of FTs we can conclude that tightly focusing the excitation beam its spatial frequency spectrum will broaden and hence stronger non-local responses are expected. Hence, analogous to the demonstrated macroscopic multipolar effects, also microscopic multipolar effects are predicted to occur solely due to the vector excitation.

The multiplicative form also provides straightforward means to engineering the NLO emission of some artificially fabricated NLO material. Provided that one could by nanofabrication affect the form of the susceptibility $\chi^{(2)}\left(\mathbf{k} ; \mathbf{k}_{1}, \mathbf{k}_{2}\right)$ and understand how it manifests for different samples ${ }^{24}$, one could design some ideal target emission properties, such as directionality, direction or beam shape, and then by some iterative algorithms solve the required $\chi^{(2)}\left(\mathbf{k} ; \mathbf{k}_{1}, \mathbf{k}_{2}\right)$. With the recent advances in fabrication of plasmonic NLO nanostructures ${ }^{25}$, this could be possible in plasmonic array structures $^{26}$.

Finally, the computational cost to calculate $\widehat{\mathbf{P}}(\mathbf{k})$ or $\mathbf{P}(\mathbf{r})$ using fast Fourier transforms (FFTs) is considerably reduced compared to brute force evaluation of the convolution integral ${ }^{27}$. In addition, the FTs of the excitation fields need to be calculated only once reducing the number of needed FTs. Therefore, the approach might provide useful for developing NLO simulation tools, where microscopic multipolar effects could also be taken into account. 


\section{CONCLUSION}

We have demonstrated how effective multipolar effects can arise in the electric dipole approximation. The effects arise when the vector nature of excitation fields are properly considered, and therefore effects become stronger when the excitation is more strongly confined. To increase the strength of the effects, we measure second-harmonic generation (SHG) using tightly focused Gaussian beams for excitation and measure strongly asymmetric transmitted and reflected SHG (5-fold difference) from a thin silicon nitride ( $\mathrm{SiN}$ ) sample. Traditional scalar treatment predicts symmetric emission. We interpret the result in terms of effective macroscopic multipolar effects, which due to the vectorial excitation. We propose an approach how higher-order Gaussian beams could be used to engineer the demonstrated multipolar effects even in the weak focusing regime. Finally, we generalize our treatment to microscopic description of light-matter interactions and propose that analogous microscopic multipolar effects could also occur for samples closer to point-like sources. Our results could provide tools to design confined and thin nonlinear sources with desired radiation properties.

\section{REFERENCES}

[1] Jackson, J. D., [Classical Electrodynamics], John Wiley \& sons Inc., New York, (1999).

[2] Barron, L. D., [Molecular Light Scattering and Optical Activity], Cambridge University Press, Cambridge, (2004).

[3] Oldenburg, S. J., Hale, G. D., Jackson, J. B. and Halas, N. J., "Light scattering from dipole and quadrupole nanoshell antennas," Appl. Phys. Lett. 75, 1063 (1999).

[4] Kelly, S. J., Coronado, E., Zhao, L. L. and Schatz, G. C., "The Optical Properties of Metal Nanoparticles: The Influence of Size, Shape, and Dielectric Environment," J. Phys. Chem. B, 107, 668 (2003).

[5] Dadap, J. I., Shan, J. and Heinz, T. F., "Theory of optical second-harmonic generation from a sphere of centrosymmetric material: small-particle limit," J. Opt. Soc. Am. B, 21, 1328-1347 (2004).

[6] Pavlyukh, Y. and Hübner, W., "Nonlinear Mie scattering from spherical particles," Phys. Rev. B, 70, 245434 (2004).

[7] de Beer, A. G. F. and Roke, S., "Nonlinear Mie theory for second-harmonic and sum-frequency scattering," Phys. Rev. B, 79, 155420 (2009).

[8] Roke, S. and Gonella, G., "Nonlinear Light Scattering and Spectroscopy of Particles and Droplets in Liquids," Annu. Rev. Phys. Chem., 63, 353-378 (2011).

[9] Curto, A. G., Volpe, G., Taminiau, T. H., Kreuzer, M. P., Quidant, R. and van Hulst, N. F., "Unidirectional emission of a quantum dot coupled to a nanoantenna," Science, 20, 930-933 (2010).

[10] Hancu, I. M., Curto, A. G., Castro-Lopez, M., Kuttge, M. and van Hulst, N. F. "Multipolar interference for directed light emission," Nano Lett., 14, 166-171 (2013).

[11]Boyd, R. W., [Nonlinear Optics], Academic Press, San Diego, (2008).

[12] Shen, Y. R., "Surface properties probed by second-harmonic and sum-frequency generation," Nature, 337, 519525 (1989).

[13]Figliozzi, P., Sun, L., Jiang, Y., Matlis, N., Mattern, B., Downer, M. C., Withrow, S. P., White, C. W., Mochán, W. L. and Mendoza, B. S., "Single-Beam and Enhanced Two-Beam Second-Harmonic Generation from Silicon Nanocrystals by Use of Spatially Inhomogeneous Femtosecond Pulses," Phys. Rev. Lett., 94, 047401 (2005).

[14] Cattaneo, S. and Kauranen, M., "Polarization-based identification of bulk contributions in surface nonlinear optics," Phys. Rev. B, 72, 033412 (2005).

[15] Shen, Y. R., [The Principles of Nonlinear Optics], John Wiley \& sons Inc., New Jersey, (2003).

[16] Boyd, G. D., "Parametric Interaction of Focused Gaussian Light Beams," J. Appl. Phys., 39, 3597 (1968).

[17] Carrasco, S., Saleh, B. E. A., Teich, M. C. and Fourkas, J. T., "Second- and third-harmonic generation with vector Gaussian beams," J. Opt. Soc. Am. B, 23, 2134 (2006).

[18] Huttunen, M. J., Mäkitalo, J. Bautista, G. and Kauranen, M. "Multipolar second-harmonic emission with focused Gaussian beams," New J. Phys., 14, 113005 (2012).

[19] Novotny, L. and Hecht, B. [Principles of Nano-Optics], Cambridge University Press, New York, (2006).

[20]Zauderer, E., "Complex argument Hermite-Gaussian and Laguerre-Gaussian beams," J. Opt. Soc. Am. A, 3(4), 465-469 (1986). 
[21] Kujala, S., Canfield, B. K., Kauranen, M., Svirko, Y. and Turunen, J., "Multipole Interference in the SecondHarmonic Optical Radiation from Gold Nanoparticles," Phys. Rev. Lett., 98, 167403 (2007).

[22] Wang, F. X, Rodríquez, F. J., Albers, W. M., Ahorinta, R., Sipe, J. E., and Kauranen, M., "Surface and bulk contributions to the second-order nonlinear optical response of a gold film," Phys. Rev. B, 80, 233402 (2009).

[23] Huttunen, M. J., Erkintalo, M., and Kauranen, M., "Absolute nonlinear optical probes of surface chirality," " J. Opt. A: Pure Appl. Op., 11, 034006 (2009).

[24] Ayoub, M., Imbrock, J., and Denz, C., "Second-harmonic generation in multi-domain chi(2) media: from disorder to order," Opt. Express, 19, 11340-11354 (2011).

[25] Kauranen, M. and Zayats, A. V., "Nonlinear plasmonics," Nat. Photonics., 6, 737-748 (2012).

[26] Czaplicki, R., Husu, H., Siikanen, R., Mäkitalo, J., Kauranen, M., Laukkanen, J., Lehtolahti, J. and Kuittinen, M., "Enhancement of Second-Harmonic Generation from Metal Nanoparticles by Passive Elements," Phys. Rev. Lett., 110, 093902 (2013).

[27]Frigo, M. and Johnson, S. G., "The design and implementation of FFTW3," Proceedings of the IEEE, 93, 216231 (2005). 